tempting its removal was questioned. An opening was torn through the gastrocolic omentum and the tumor wall exposed; it had a dark bluish color resembling a cyst.

It was now diagnosed as a pancreatic cyst and I decided on immediate incision and drainage. The edges of the omentum, which was firmly adherent to the cyst wall, were stitched to the peritoneum of the abdominal wound in order to prevent leakage into the abdominal cavity. An incision 1 inch long was now made into the cyst and its contents drained out by turning the patient on her left side. The fluid contents, a dark greenishyellow color and quite turbid, had a sourish odor. Unfortunately none was preserved for analysis. The quantity was estimated at 2000 c.c. It was not possible to reach the upper end of the sac with the finger inserted in the opening. A probe passed upward, backward and outward for about $25 \mathrm{~cm}$. toward the tail of the pancreas.

The edges of the cyst wall, which were quite thick, were sutured to the abdominal wound and a large rubber drainage-tube inserted into it. The external wound was closed with silkworm gut sutures and an absorbent dressing applied.

The shock of the two operations was quite severe, and the patient's temperature remained subnormal for the first twelve hours. She was very restless and suffered severe pain after the effects of the anesthetic wore off. She was stimulated by morphia and strychnia hypodermically, together with hot saline enemas. The pains continued more or less severe for four days, and the nausea following the anesthesia lasted for threedays.

The discharge from the drainage-tube was very slight after the second day and the tube was removed on the eighth day. The wound was entirely closed on the eighteenth day. The patient gained strength very rapidly as soon as able to take nourishment, and her recovery was uneventful. At the end of six months her health is good and she is able to do her own housework.

\section{SMALLPOX IN OHIO.*}

BY C. O. PROBST, MLD.

SECRETARY OHIO STATE BOARD OF HEALTH. COLUMBUS, OHIO.

Ohio has been having a most interesting epidemic of smallpox during the past year, and I had hoped to he able to present a proper report of it to this meeting. During the past six or eight months, however, I have been kept so busy combatting the disease that I have had no opportunity to complete the report. A few weeks ago I wrote the Chairman that I would be unable to present the paper I had promised, but he informed me that the program had already been printed and that he would expect me to say something on the subject. I have, therefore, very hurriedly prepared a few remarks on some of the more interesting features of our epidemic.

Smallpox made its appearance in Ohio April 6, 1898, and up to June 6, 1899, fourteen months, there have been reported $188^{\circ}$ cases and 30 deaths. Out of 88 counties 45 have been invaded, and the disease has prevailed in 61 cities and villages, besides, in most instances, invading rural districts surrounding them. The disease has been marked by its exceeding mildness and by the many failures to recognize its character, both operating to favor its spread.

* Presented to the Section on State Medicine, at the Fiftieth Annual Meeting of the American Medical Assoclation, held at Columbus, Ohlo, June 6-9, 1899.
The first outbreak occurred in a small village a few miles south of this city and very well illustrates the nature of the disease. A man 72 years old made a visit South, passing through several States where smallpox was prevailing. About a week after returning home he was taken ill and in a few days died with what was called "black measles." Two weeks later his wife, son, and three grandchildren were attacked with what was pronounced "chicken-pox." The children had never been vaccinated, and yet the disease was exceedingly mild, so mild that the children were never bedfast and scarcely missed a meal. The eruption was scanty, rapidly ran its course, and presented much the appearance of chicken-pox. Two or three well-marked cases of smallpox resulted from exposure to these children and settled the diagnosis.

In April, 1898, a' show known as "Uncle Tom's Cabin Show," owned in Columbus, started on its annual tour, which for that year was at first through the western counties of Ohio. Two children, one of them "Topsy," and an adult contracted what physicians called to see the patients pronounced "chicken-pox." The children were not greatly affected at any time, Topsy taking her part in the play each night. 'The adult was quite ill for some time. Six distinct outbreaks in as many different communities were directly traced to this show. A most remarkable feature of these outbreaks is that in none of them was the nature of the disease thus started recognized until months afterward.

One of these communities, where smallpox was thus started, is Wapakoneta. The disease began there in May, and it was not until October that an investigation was made, or called for, and the disease declared "smallpox." The community was largely unvaccinated, most of the children under 12 years of age being unprotected. No restricted measures whatever were employed. Schools and public gatherings continued as usual. The sick were visited and every opportunity was afforded for the spread of the disease. A county fair was held and smallpox patients in the desquamative stage of the disease mingled freely with the visitors. In spite of this great exposure there was comparatively little spread of the disease. In all there were but 203 cases of smallpox in Wapakoneta and vicinity, embracing a population of not less than 9000 people. The great majority of these occurred before preventive measures of any kind were employed.

Perhaps a more remarkable behavior of the disease was shown in Marysville, a village of about 4000 inhabitants. Smallpox appeared there in May, the first three cases having been directly traced to exposure to the troupe referred to above. The disease spread very slowly, attacking children and adults, but only the unvaccinated. The health officer, some time in July or August, became suspicious of smallpox and called a council of physicians. They agreed on a diagnosis of "impetigo contagiosa." No efforts were made to prevent the disease from spreading, the diagnosis of impetigo being accepted, the common people, however, calling it "Spanish measles."

An outbreak of smallpox in Hamilton the 1st of November was directly traced to an importation from Marysville. Inquiry developed the fact, however, that at that time there were no cases of either smallpox or impetigo in Marysville. The disease had actually died out of its own accord.

Two weeks later the health officer of Marysville reported some suspicious cases, and three undoubted cases of smallpox were found in one family. These were quarantined and no others occurred. Probably most of 
the physicians in Marysville will still tell you that there has not been a case of smallpox in that village.

These are fair examples of numerous outbreaks in different communities of this state, where smallpox has languished for weeks and months without any one suspecting its presence.

'The disease itself has presented many unusual features. It has been not only very mild, but also very feebly contagious. What has been said would indicate this, but other facts show it. In some instances unvaccinated children have lived in the house where persons went through with an attack of smallpox, associating with them daily, and yet escaped the disease. Persons with the diseasc have gone into crowded rooms, as into schools, churches, railway cars, etc., and yet no one contracted smallpox from such exposure. Vaccination, even if performed many years ago, has given almost perfect protection.

Where the disease has prevailed in a community for months, masked under some other name, vaccination became the best means for diagnosis. A house-to-house canvass in such cases, with a history of the patients and their families, revealed the fact that with few exceptions the disease had gone through family after family, picking out only the unvaccinated. There was a remarkable instance of this at Marysville. Two families living in adjoining houses in the same yard had the disease. In one family of seven all had smallpox and all were unvaccinated. In the neighboring family of six but one contracted the disease and he was the only unvaccinated member of this family.

The symptoms of the disease were variable. The preliminary fever was in many cases slight and almost unnoticed. Secondary fever appeared in only a small proportion. The eruption in most instances was more superficial than common, involving the true skin less, so that in most cases the pitting was slight. The eruptive period was often much shortened and pursued a somewhat irregular course. While the disease was so very mild several deaths, nevertheless, occurred from hemorrhagic smallpox, and two from the malignant, purpuric form.

In conclusion may be quoted what an old, experienced phrsician in Wapakoneta said when the character of the diccase there was being investigated: "If this disease we are having here is smallpox then the whole subject of smallpox, as given in our text-books, must be rewritten." But when asked if that would not be equally true if the disease were chicken-pox, as it was being called, he said: "I guess that's so, too."

For discussion. see page 1592 .

\section{HOW SHALL WE CONTROL VARIOLA?* BY S. I. JEPSON, A.M., M.D. \\ CJTY HEALTH OFFICER. WHFELING, W, va.}

A pebble in the streamlet scant

Has changed the course of many a river.

Vigorous sanitation applied at the right time and in the right way will always prevent an epidemic of smallpox. No acute infectious disease is so easily controlled, because: 1. It manifests itself externally and should rarely be mistaker for any other disease; 2 , it is greatly dreaded, and hence people are as a rule careful to avoid it; 3 , its germs re easily destroyed; 4 , we have in vaccination an absolutely certain protection. The last state-

* Presented to the Section on State Medicine, at the Fiftieth Annual Meeting of the American Medical Association, held at Columbus, Ohio. June 6.9, 1899. ment may be disputed, but the exceptions are so very rare that I think better to ignore them.

My experience as a sanitary officer has brought to light a very great amount of prejudice against vaccination. This is largely based on the densest ignorance, but not a small part of it is due to the many bad results of careless vaccination-results often caused, not by impure virus, but'by vicious methods of introduction or neglect by physicians of the commonest antiseptic precautions. Arms are seldom cleaned prior to the operation. Not rarely absorbent cotton or a cotton rag is bound tightly over the arm; and at least two physicians whom I know apply adhesive plaster after the insertion of the virus, allowing it to remain during the development of the vesicle. The result is a broken vesicle or pustule, an imperfect protection, often an infected and consequently very sore arm, with constitutional disturbance; and, worse than all, a growing prejudice against this most beneficent of all known preventives of disease.

The past year has given several demonstrations of the carelessness, ignorance, or perversity of physicians that are by no means creditable to our profession. In the very enlightened states of Ohio and Pennsylvania have errors been made in the diagnosis of variola and persistently adhered to until the disease spread far beyond the limits of its origin. No man is infallible, and some unusual forms of variola, as the malignant, the hemorrhagic, and some cases greatly modified by vaccination, may mislead for a time the most experienced. But to persist in error in opposition to more enlightened and expert opinions, and thus allow a disease to spread which ruins the business of a community and entails upon it a large expenditure of money, to say nothing of the resulting inconvenience, suffering, disfigurement, and death, is little short of criminal.

When a disease is so widely prevalent as smallpox has been for several years past, physicians should keep it in mind, and when called to strangers or those who may have been in an infected locality, they would do well to ask themselves the question: "Is this a case of smallpox?" Until this question can be definitely answered in the negative, let the case be isolated and every sanitary precaution be taken. Better quarantine a hundred cases of varicella than to run any risk of starting a smallpox epidemic.

Little less culpable than persistent errors in diagnosis are long delays in making a diagnosis, unless the strictest sanitary precautions are observed while awaiting developments. Some physicians never report a case of variola, even. when it is prevailing in a community, until the second or third day of the eruption, free intercourse with the patient being allowed in the meantime, with the result of communicating the disease to one or more persons who, under proper management, would have escaped it. While such a practice is absolutely without excuse, personal experience has taught me that it is by no means uncommon.

The facts here recited lead me to impress these suggestions on the general practitioner:

1. Insist on general vaccination and use your influence in having the best virus inserted with all antiseptic precantions. I use the glycerinized in closed glass tubes.

2. Try to avoid errors in diagnosis. If in doubt, call to your aid the proper health officer or some one more familiar than yourself with smallpox. Do not tenaciously adhere to an opinion that the case is varicella if an adverse opinion is expressed by a physician of larger experience with variola than is your own. The 